\title{
SUSTENTABILIDADE NO USO DO BIODIESEL: modelagem computacional baseada em dinâmica de sistemas
}

Glauco Oliveira RODRIGUES ${ }^{1}$

Eugênio de Oliveira SIMONETTO²

Felipe RAVANELLO 3

Gabriela BELTRAME ${ }^{4}$

Francies Diego MOTKE 5

\author{
${ }^{1}$ Programa de Pós-Graduação em Administração- PPGA,-CTISM, glaucop10@ redes.ufsm.br \\ Universidade Federal de Santa Maria - UFSM - Santa Maria/RS - Brasil \\ ${ }^{2}$ Programa de Pós-Graduação em Administração- PPGA, eosimonetto@gmail.com \\ Universidade Federal de Santa Maria - UFSM - Santa Maria/RS - Brasil \\ ${ }^{3}$ Programa de Pós-Graduação em Administração- PPGA, feliperavas@ gmail.com \\ Universidade Federal de Santa Maria - UFSM - Santa Maria/RS - Brasil \\ ${ }^{4}$ Programa de Pós-Graduação em Administração- PPGA, gabibeltrame @ hotmail.com \\ Universidade Federal de Santa Maria - UFSM - Santa Maria/RS - Brasil \\ ${ }^{5}$ Programa de Pós-Graduação em Administração- PPGA, fdmotke@gmail.com \\ Universidade Federal de Santa Maria - UFSM - Santa Maria/RS - Brasil
}

Recebido em: 22/01/2016 - Aprovado em: 23/06/2016 - Disponibilizado em: 30/07/2016

\begin{abstract}
Resumo
Este artigo apresenta uma proposta de modelo de simulação para diminuir os gastos, poluição da água e emissão de CO2, relativos a coleta de resíduos sólidos urbanos na região central do estado do Rio Grande do Sul. Será utilizado diversas porcentagens de biodiesel, que junto a porcentagens de diesel formará um combustível com menor impacto ao meio ambiente. Para a definição de variáveis e suas inter-relações, utilizou-se, por base teórica as pesquisas bibliográficas e observações do processo da geração dos resíduos pós-uso do óleo de cozinha. Para avaliar a possibilidade de geração de um novo combustível, que poderá ser utilizado pelos veículos coletores de resíduos sólidos dos municípios da região central do estado, foram gerados três cenários, com o objetivo de simular 3 misturas de biodiesel. Os resultados obtidos através da simulação demonstram que além da grande redução do impacto ambiental, o processo de reciclagem traz um significativo ganho econômico em função da redução de custos com a aquisição de combustível. O horizonte de tempo simulado foi de 10 (dez) anos e foi utilizado o software Vensim para o desenvolvimento da simulação.
\end{abstract}

Palavras-chave: Biodiesel. Modelagem Computacional. Sustentabilidade.

\begin{abstract}
This article presents a simulation model proposed to reduce spending, water pollution and $\mathrm{CO} 2$ emissions, for the collection of municipal solid waste in the central region of Rio Grande do Sul state. It will be used several biodiesel percentages, which together with Diesel percentages form a fuel with less impact to the environment. For the definition of variables and their interrelationships, it was used for theoretical basis bibliographical research and observations of the generation of post-use waste cooking oil process. To evaluate the possibility of generating a new fuel that can be used by collecting vehicles of solid waste in the municipalities of the central region of the state, three scenarios were generated in order to simulate three biodiesel blends. The results obtained by simulation show that in addition to the large reduction of the environmental impact, the recycling process brings significant economic gain due to the reduction of costs for the purchase of fuel. The horizon of simulated time was ten (10) years and was used Vensim software for the development of simulation.
\end{abstract}

Keywords: Biodiesel. Computational modeling. Sustainability. 


\section{Introdução}

A preocupação que vem recebendo grande atenção é a destinação final do lixo, devido a toda a sua implicação ambiental que afeta a qualidade de vida da população. No Brasil, assim como em outros países, a preocupação com a destinação final adequada dos resíduos sólidos vem sendo muito discutida. Segundo Oliveira e Sommerlatte (2009), essa destinação é um dos maiores problemas da sociedade moderna, pelo fato da composição dos resíduos urbanos estarem modificado-se ao longo dos últimos anos e a sua produção aumentou significativamente.

Apesar de toda a preocupação com a preservação do meio ambiente, o que acontece na prática, na maioria das vezes, é um desenvolvimento não-sustentável, já que a coleta, o transporte e o depósito desses resíduos não levam em conta um tratamento adequado. Dentre esses resíduos sólidos, destaca-se o óleo de cozinha, de acordo com a Associação Brasileira das Indústrias de Óleo Vegetal, são produzidos aproximadamente cerca de 3 bilhões de litros de óleo por ano no Brasil, mas apenas 2,5\% de todo esse óleo é reciclado e reutilizado na cadeia produtiva.

Ao mesmo tempo que o óleo de cozinha é um produto nocivo ao meio ambiente, quando descartado de forma inadequado, é também um excelente subproduto para a cadeira produtiva (OLIVEIRA ; SOMMERLATTE,
2009). Sob esse enfoque os óleos residuais de frituras representam um grande potencial de oferta desse insumo. Essa matéria-prima pode ser adquirida em lanchonetes e cozinhas industriais, empresas onde ocorre a fritura de produtos alimentícios, em esgotos onde a nata sobrenadante é rica em matéria graxa e águas residuais de processos de indústrias alimentícias. Assim, a reciclagem dos resíduos sólidos, como o óleo de cozinha, gera economia de energia, gera empregos e aumenta a conscientização da população para as questões ambientais. Segundo o Ministério do Meio Ambiente, desde agosto de 2010, baseado no conceito de responsabilidade compartilhada, cidadãos, governos, setor privado e sociedade civil, passaram a ser responsável pela gestão ambientalmente adequada dos resíduos sólidos. O cidadão é responsável não só pela disposição correta dos resíduos que gera, mas também é importante que repense e reveja o seu papel como consumidor. O setor privado, fica responsável pelo gerenciamento ambientalmente correto dos resíduos sólidos, pela sua reincorporação na cadeia produtiva e pelas inovações nos produtos que tragam benefícios socioambientais, os governos federais, estaduais e municipais são responsáveis pela elaboração e implementação dos planos de gestão de resíduos sólidos, assim como dos demais instrumentos previstos na PNRS. Dentre estes, destaca-se os Planos de Gestão 
Integrada de Resíduos Sólidos Urbanos, por ser uma nova alternativa para abordar a sustentabilidade comprometida com a proteção ambiental. Essa alternativa caracteriza e quantifica os resíduos sólidos gerados, obtendo assim, serviços com uma melhor qualidade, custos reduzidos e ações que incentivam a redução, a reciclagem e o reaproveitamento.

Nesse cenário, por ser um excelente subproduto, o óleo de cozinha pós-utilização pode receber um destino correto por meio do seu reaproveitamento e reciclagem. $\mathrm{O}$ mesmo pode ser usado na produção de sabão, detergentes, ração animal, resina para colas, assim como na produção de biodiesel, que tem se mostrado uma alternativa para os combustíveis fósseis, o mesmo utiliza matéria-prima renovável, agride menos o meio ambiente, reduzindo a emissão de gases causadores do efeito estufa (GEE, 2015). A produção de um Biocombustível a partir do óleo de cozinha proporcionaria inúmeros benefícios para a sociedade, como a diminuição de vários problemas relacionados ao seu descarte, mas além destes benefícios, ainda haveria a possibilidade de aumentar a produção e a utilização de Biocombustível, como no caso o Biodiesel, contribuindo com o meio ambiente através da diminuição da emissão de gases de efeito estufa (BARBOSA; PASQUALETTO, 2006. Na perspectiva de Oliveira, Suarez e Santos (2008), o uso de combustíveis alternativos vem ganhando espaço, já que o petróleo é uma fonte esgotável de energia e gera grande quantidade de gases poluentes.

Diante desse contexto, o presente trabalho apresenta a proposta de um modelo de reaproveitamento do óleo de cozinha, para a geração de biodiesel que substitua totalmente ou parcialmente o diesel utilizado nos caminhões de coleta de lixo de Santa Maria. Nos próximo item, 2 , será apresentada a metodologia utilizada na realização do trabalho e nas suas subseções o referencial teórico. Na sequência, no item número 3, será apresentado o modelo computacional desenvolvido e suas variáveis. No item 4 serão abordados os principais resultados obtidos com o estudo, a validação do modelo é apresentada na seção 5 e, por fim, será apresentada a conclusão.

\section{Método de Pesquisa}

Neste trabalho o método de pesquisa adotado para o desenvolvimento do modelo computacional foi baseado na metodologia criada por Law e Kelton (1991), a qual é constituída pelos seguintes passos: (1) estudos exploratórios em artigos científicos, relatórios e entrevistas com os gestores do ambiente simulado, no caso uma empresa de coleta de óleo queimado e uma empresa de recolhimento de lixo de municípios da re região central do estado do Rio Grande do 
Sul. Através desses dados o problema foi especificado e estruturado;

desenvolvimento da solução, pela construção de modelos formais capazes de representar o problema; (3) implementação computacional da solução, utilizando-se o simulador Vensim da área de system dynamics; (4) validação da solução, através de testes em laboratório e análise do comportamento histórico, para verificar se os resultados obtidos representam a realidade observada, bem como através da simulação de um experimento utilizando dois cenários para tal. Também foram entrevistados gestores das áreas envolvidas para garantir uma maior fidedignidade ao estudo.

\subsection{Biodiesel}

O Biodiesel é visto por muitos pesquisadores como uma fonte de energia secular, que possui um crescimento recente. Dois motivos principais fazem com que este crescimento seja continuo: aumento do valor do barril do petróleo e preocupações ambientais. É um combustível obtido a partir de matériasprimas vegetais ou animais. As matériasprimas vegetais são derivadas de óleos vegetais, tais como soja, mamona, palma (dendê), girassol, pinhão manso, amendoim, entre outros (TOPPAN; DARBELLO, 2009). As matérias-primas de origem animal podem ser obtidas do sebo suíno, bovino e de aves, também pode ser obtido utilizando como matéria-prima, óleos utilizados em fritura (cocção). Santos e Matal (2006) destacam o biodiesel, entre as fontes de energia renovável,como uma solução para minimizar alguns problemas ambientes decorrentes do uso de combustíveis derivados do petróleo, redução de hidrocarbonetos, monóxido de carbono e redução na emissão de dióxido de carbono são alguns dos benefícios ambientais da utilização do biodiesel em motores.

\subsection{O uso do Biodiesel em motores de combustão}

Em misturas de óleo diesel e biodiesel em proporção de até $10 \%$ ocorreu uma redução no consumo de combustível, e para proporções maiores que essa ocorreu um aumento no consumo, chegando a $4,77 \%$ quando utilizado o biodiesel puro.Esse aumento no consumo é justificado pela diferença no poder calorífico do biodiesel, que em geral se apresenta menor que o poder calorífico do óleo diesel.

Agarwal e Das (2001) verificaram que o uso do combustível B20, testado em um motor a diesel, teve o melhor desempenho entre todas as misturas analisadas, com um benefício de 2,5\% na eficiência térmica máxima e uma redução significativa nos teores de fumaça. Dorado (2002) concluíram que o motor a diesel, analisado sem nenhuma modificação, 
funcionou de maneira satisfatória com misturas de $10 \%$ de biodiesel de óleo de fritura e $90 \%$ de óleo diesel.

Ferrari, Oliveira e Seabio (2005) realizaram testes utilizando $5 \%$ de biodiesel e $95 \%$ de diesel convencional (B5) durante um ano, tendo percorrido $19.240 \mathrm{~km}$ em condições normais de trabalho, e observaram que o veículo apresentou desempenho normal com redução da emissão de fumaça. Além disso, durante o período de realização dos testes não foram necessários reparos no motor, o que induziu os pesquisadores a concluir que os óleos vegetais transesterificados se adaptam perfeitamente ao motor.

\subsection{Critérios de Sustentabilidade e} Biodiesel

Segundo Mendes (2015), não é só ao meio ambiente que está relacionada a sustentabilidade, ela possui um grande foco na análise da questão econômica e social.

Conseqüentemente deve-se medir os impactos gerados ao meio ambiente e aos custos relacionados na produção e uso do biodiesel, para verificar se este combustível é mais sustentável que o Diesel, verificando também as diferenças dos ganhos sociais e o que cada um pode oferecer ainda a sociedade mundial. Um modo de verificar e medir estes impactos citados a cima, é a Análise do Ciclo de Vida (ACV). Através desta análise pode- se verificar estes impactos, desde sua criação passando pelas etapas de produção,distribuição e o uso final deste produto.

\section{O Modelo de System Dynamics}

Como afirma Dace, Bazbauers, Berzina e Davidsen (2014), na década de 50 o engenheiro Jay Forrester criou a metodologia de System Dynamics, aplicando inicialmente na verificação das oscilações nas vendas em uma empresa da América.

System Dynamics (SD) permite o estudo do comportamento dos sistemas ao longo do tempo, permitindo a avaliação das consequências de nossas decisões. Por essa razão e a necessidade de estudar os impactos da reciclagem dos resíduos em um horizonte temporal futuro, decidiu-se utilizá-la na modelagem e simulação computacional. Um modelo de SD pode ser definido como a estrutura resultante da interação de políticas. Esta estrutura é formada por dois componentes principais, que são os estoques e os fluxos. Ford (2009) define os SD como uma combinação de estoques e fluxos que utilizam uma estrutura computacional para serem simulados.

Um modelo computacional tem como característica sua transparência, permitindo a criação de modelos complexos caracterizados por atrasos e mecanismos de feedback que 
apresentam explicitamente as relações de causa e efeito do modelo estudado. A analise do System Dynamics é realizada, alterando os valores dos parâmetros individualmente ou em combinações das várias compostas do modelo, através destes parâmetros nota-se o comportamento do sistema. Quatro componentes constituem um modelo de System Dynamics.

Inicialmente temos os estoques que são variáveis de estado e podem ser considerados como repositórios para acumular ou armazenar outros elementos do sistema (DEATON; WINEBRAKE, 2000). Por sua vez, os fluxos, são variáveis de ação, elas podem aumentar ou diminuir o volume determinado no estoque. Para formular os dados, definem-se equações dos fluxos, combinadas através de operações algébricas, existem os auxiliares que também podem modificar outras variáveis auxiliares, são muito utilizadas para modelar as informações e não o fluxo físico, podendo alterar instantaneamente sem atrasos (COVER, 1996). Por fim existem os conectores que são os que representam as inter-relações entre todos os componentes, ou seja, são essas inter-ligações que ligam os componentes que formam a expressão matemática (DEATON; WINEBRAKE, 2000). A Figura 1 demonstra cada um dos componentes de um modelo de System Dynamics.
Figura 1. Comportamento de um modelo de System Dynamics

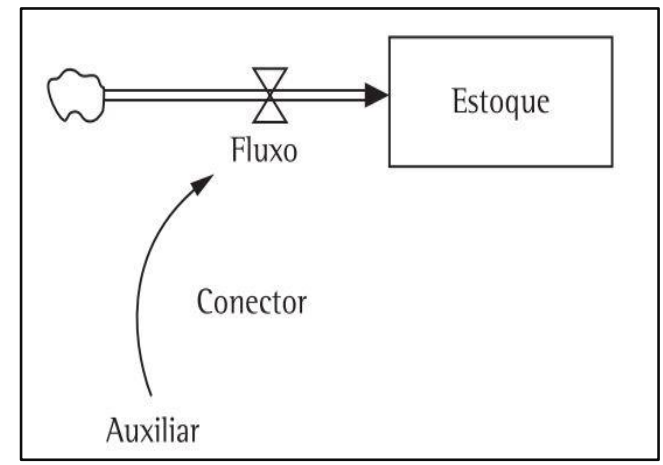

Fonte: Simonetto, E. de O.; Lobler, M. L (2013)

Para realizar a modelagem computacional deste trabalho foram coletados dados de uma empresa responsável pela coleta das cidades de São Francisco de Assis, Cacequi, Mata, São Martinho da Serra, Agudo, Restinga Seca e Faxinal, localizadas na região central do Rio Grande do Sul, toda a coleta é encaminhada para o aterro sanitário da cidade de Santa Maria. A execução das simulações foi utilizado o simulador Vensim (VENTANA SYSTEMS, 2011) em uma estação de trabalho com processador intel Core (i5) e 8 Gb de memória RAM.

\subsection{A Estrutura do Modelo}

A figura 2 demonstra a estrutura do modelo desenvolvido, com as três principais variáveis, o "modelo de coleta", "modelo de reaproveitamento" e o modelo de economia. Os mecanismos do modelo de coleta representa as equações necessárias para a realização da coleta e o transporte dos 
resíduos sólidos das cidades estudadas até o aterro situado na cidade de Santa Maria. O "modelo de reaproveitamento" possui a importância de calcular os valores referentes a transformação da materia prima, neste caso o óleo queimado, em biodiesel. Por fim, o "o modelo de economia" é responsável pela comparação de gastos entre a coleta atual e a coleta utilizando biodiesel. Veremos a seguir mais informações sobre o mesmo.

Figura 2. Estrutura do Modelo

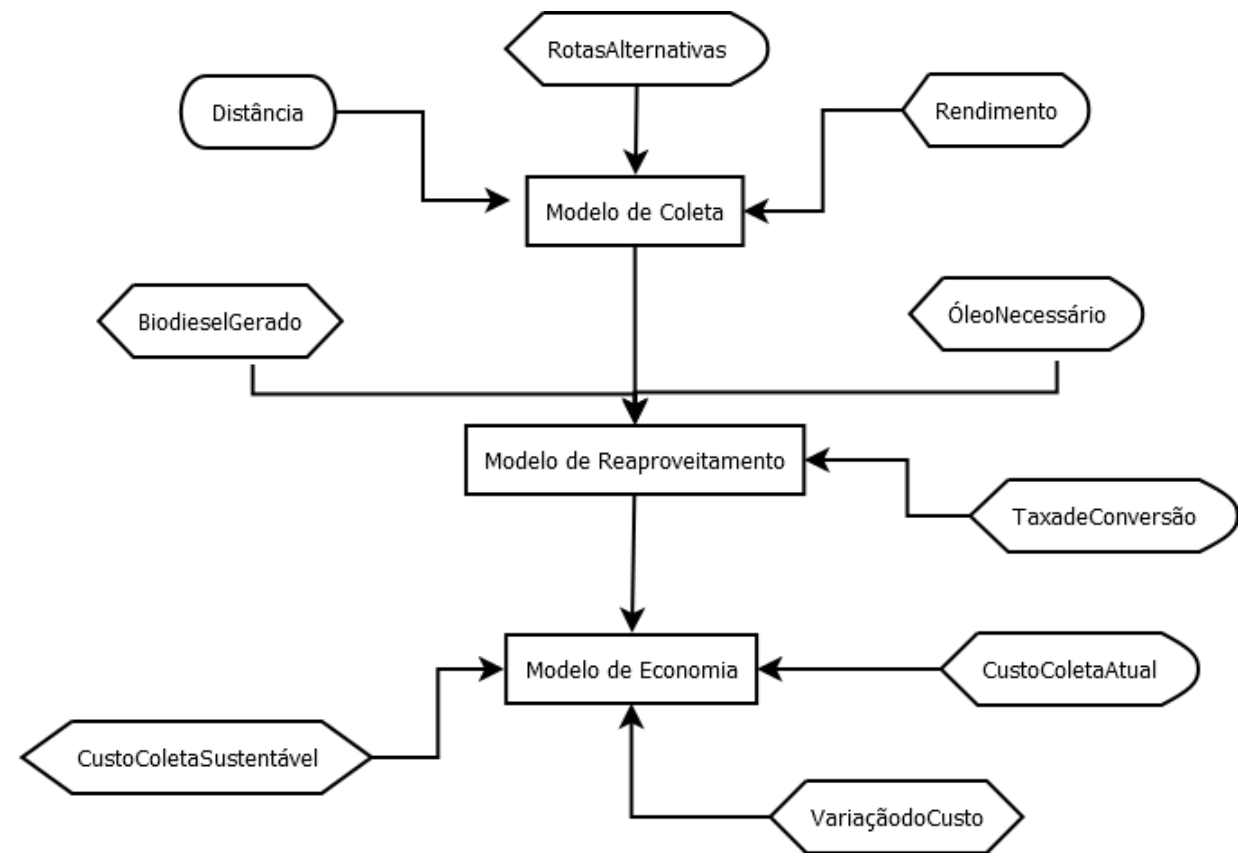

Fonte: Próprios Autores (2016)

rendimento do motor de cada caminhão de coleta.

\subsubsection{Modelo de Coleta}

O Modelo de coleta representado na figura 3 tem como objetivo principal armazenar os valores finais da coleta dos resíduos sólidos urbanos, contendo informações da distância (em $\mathrm{Km}$ ) percorrida necessária, e também o acréscimo das rotas alternativas, utilizadas em caso de desvios urbanos, junto com o
Figura 3. Modelo de Coleta

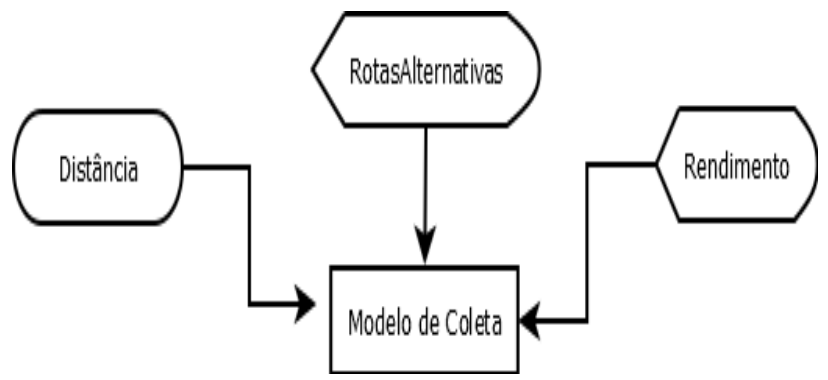

Fonte: Próprios Autores (2016) 


\subsubsection{Modelo de Reaproveitamento}

A figura 4 apresenta o modelo de Reaproveitamento, contento informações necessárias para a reutilização do óleo de cozinha, este modelo auxilia a construção de variáveis importantes para a formação do combustível mais adequando para o meio ambiente mundial. Com seu auxilio será fixada a quantia máxima de biodiesel produzido por cenário modelado. Através deste modelo, será possível comparar os gases emitidos na atmosfera mundial, verificando qual a proporção de biodiesel com um menor grau de poluição.

Figura 4. Modelo de Reaproveitamento

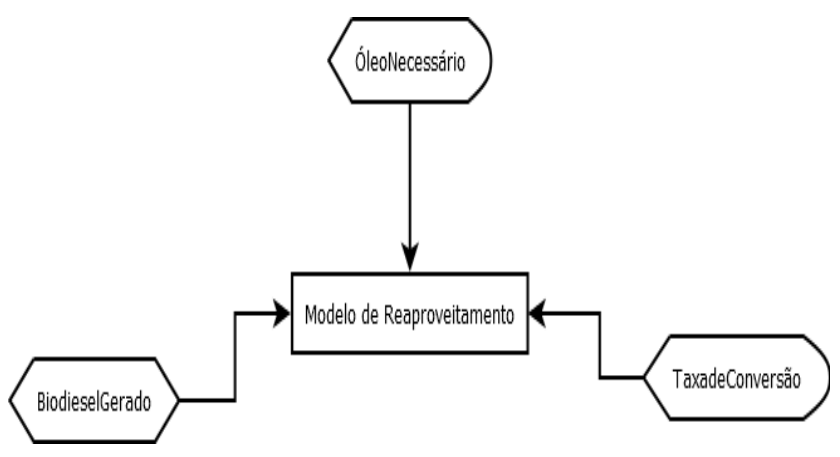

Fonte: Próprios Autores (2016)

\subsubsection{Modelo de Economia}

O objetivo do modelo de economia é comparar o gasto da coleta atual com a variação da coleta utilizando biodiesel, verificando as variações em cada cenário simulado. A figura 5 expõem as variáveis necessárias para a construção do modelo, a variação do custo conforme os anos é estudada neste artigo, é essencial verificar a exata variação das taxas, pelo fato da mesma afetar o resultado da modelagem que será realizada.

Figura 5. Modelo de Economia

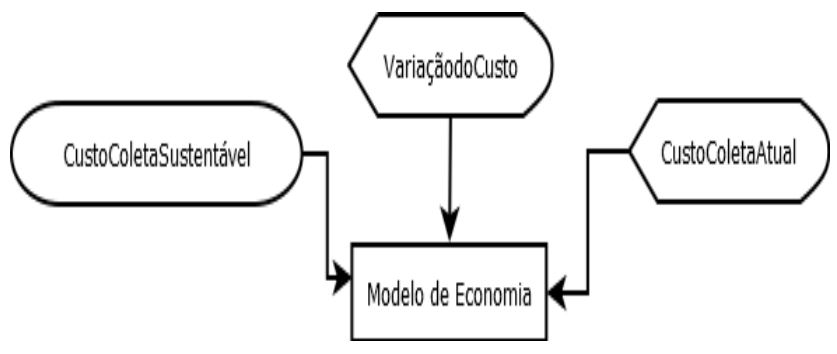

Fonte: Próprios Autores (2016)

\subsection{O Modelo Computacional Desenvolvido}

Após a construção dos 3 modelos de auxilio, tendo por embasamento a importância dos processos citados para a preservação do meioambiente e os ganhos ambientais ocasionados por esses, desenvolveu-se um modelo de simulação, o qual permitirá tanto aos gestores ambientais, quanto aos da área de resíduos avaliarem políticas de reciclagem/reaproveitamento do óleo de cozinha, onde avaliou- se os ganhos ambientais, visando o desenvolvimento sustentável gerados por este, bem como os benefícios econômicos do processo.

No modelo os ganhos ambientais que serão avaliados futuramente, serão a redução da poluição da água e da emissão de $\mathrm{CO} 2$. Com 
relação aos ganhos financeiros, a redução de gastos com a coleta total na região central do estado. O modelo foi desenvolvido buscando simplificar a interação usuário-computador, para que análises do tipo "o que se?" (whatif?), comuns em modelos de simulação, sejam de rápida e simples execução.

Para a definição das variáveis do modelo de simulação, foram realizadas entrevistas com especialistas da área, onde verificou toda a Quadro 1. Modelo de equações.

OleoParaReciclar $=$ TotalColeta

ReduicaoPoluicaoAgua $=18400 *$ OleoParaReciclar

DieselTotal $=$ DieselPorAno

BiodieselGerado $=$ OleoParaReciclar $*$ TaxaAprovOleo

GeracaoCO2 $=(2.669 *$ DieselTotal $)-(2.669 * 0.85) *$ BiodieselGerado

Economia $=(($ ConsumoMedioBiodiesel $*$ BiodieselGerado $)$

(CustoDiesel*DieselTotal)/ConsumoMedioDiesel)-CustoBiodiesel

Fonte: Próprios Autores (2016)

A variável OleoParaReciclar obtida a partir da variável totalcoleta, é a variável que possui a quantidade de óleo coletado durante os 10 anos com as variações necessárias para produzir o biodiesel desejado, ela será representadas na equação (1) do modelo de equações do quadro1;

A variável ReducaoPoluicaoAgua é obtida através do produto da quantidade de óleo residual pelo potencial poluidor de cada litro despejado inadequadamente, ou seja, 18400 litros de água por litro de óleo. A variável é descrita na equação (2) do modelo de equações apresentado no Quadro1. sistemática do problema estudado, trabalhos acadêmicos e governamentais da área de resíduos, BNDES (2004), CONAMA (2011) e Oliveira e Filho (2014) complementaram esta etapa. As variáveis selecionadas, bem como suas inter-relações com outras variáveis, as quais influenciam nos valores totais do reaproveitamento do óleo de cozinha na produção de biodiesel e utilização como combustível são: 
2.669 pelo produto da quantidade de biodiesel gerado pela quantidade de emissão de $\mathrm{CO} 2$ por litro de diesel comum (2.66kg/CO2). A equação (6) do modelo de equações descreve a variável.

A variável Economia representa os ganhos financeiros do reaproveitamento do óleo de cozinha e é obtida utilizando-se as variáveis ConsumoMedioBiodiesel(que representa o custo estimado por litro de biodiesel produzido), BiodieselGerado, CustoDiesel e
DieselTotal. A variável é descrita na equação (7) do modelo de equações apresentado no Quadro1.

A Figura 6 apresenta o modelo computacional final, representando as interligações entre estoques e fluxos, nota-se a variável "time" conectada em 4 variáveis, é a partir destas conexões que simularemos a variação dos valores durante 10 anos.

Figura 6. Modelo de Simulação Proposto

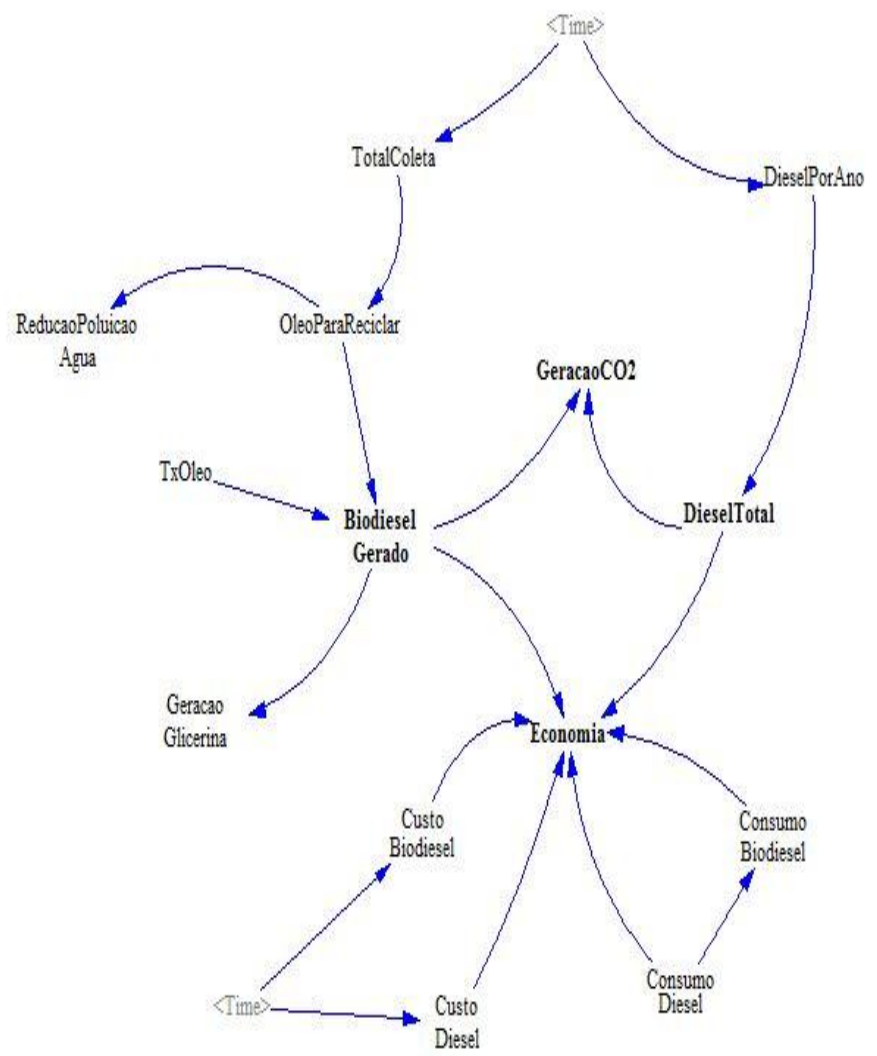

Fonte: Próprios Autores (2016)

\subsection{Análise dos Cenários}

A razão de transformação do óleo de cozinha em biodiesel, através da inserção do metanol é da ordem de $80 \%$ (OLIVEIRA ; SOMMERLATTE, 2009., Embora outras referências apresentem divergências neste valor (ZUCATTO ; WELLE ; SILVA, 2013). No Brasil, a legislação estabelece desde o ano 
de 2013, que o diesel comum deve ter presente 5\% de biodiesel, a partir desta mistura poderá ser adicionado até $20 \%$ de biodiesel ou B20, que é a mistura máxima suportada pelos motores a diesel, sem adaptação. Desta forma, para cada litro de diesel B5 deverá ser adicionado $190 \mathrm{ml}$ do biodiesel produzido. Além da economia gerada pela redução da compra de diesel para a utilização nos veículos da coleta, existem outras vantagens adicionais que poderão ser obtidas pela utilização do biodiesel, uma delas é a redução do impacto ambiental resultante do descarte inapropriado, já que a utilização do biodiesel auxilia na diminuição da emissão do dióxido de carbono na atmosfera.

De acordo com os dados da resolução do CONAMA (2011), é admitido a presença de até $50 \mathrm{mg} / \mathrm{l}$ de óleos vegetais e gorduras animais nos efluentes. A densidade recomendada pela ANVISA para o óleo de cozinha é de 0,920. A partir destes dados, pode-se calcular de fato o impacto ambiental causado por litro de óleo descartado de forma indevida no ambiente. Desta relação tem-se que cada litro de óleo precisa de 18400 litros de água para dispersão.
$\mathrm{Na}$ modelagem que será desenvolvida, a variável causadora de maior impacto a sensibilidade do modelo é a Economia. Essa é diretamente afetada por variações na coleta de resíduos, já que será feito um estudo de custo da coleta durante 10 anos, variando as taxas tanto de quantidade de diesel e quantidade de biodiesel utilizado na coleta. $\mathrm{O}$ custo de produção do biodiesel é fortemente influenciado pelo custo da matéria prima utilizada. Os custos envolvidos referentes ao processo e aos demais produtos necessários a reação química são estimados em torno de $\mathrm{R} \$$ 0,30 por litro de óleo, ou seja, aproximadamente $\mathrm{R} \$ 1,05$ por litro de biocombustível B100 .

Sabe-se que o diesel comum emite 2.669 $\mathrm{Kg} / \mathrm{CO} 2$ por litro de combustível queimado, a proposta é minimizar o impacto ambiental causado pelos resíduos gerados, isso pode ser obtido através de processos mais eficientes de transformação ou através da redução de geração destes resíduos. A tabela 1 representa os valores utilizados para a simulação dos 3 cenários propostos, contendo as taxas necessárias para a viabilização da modelagem.

Tabela 1. Variáveis e valores utilizados nos Cenários

\begin{tabular}{cc}
\hline Variável & Cenário Simulados \\
\hline $\begin{array}{c}\text { Taxa de Aproveitamento } \\
\text { do óleo no processo } \\
\text { de reciclagem }\end{array}$ & $\begin{array}{c}\text { Fixada em } 80 \% . \\
\text { Custo Diesel }\end{array}$ \\
$\begin{array}{c}\text { Custo de Produção (Biodiesel) } \$ 2,89 \text { no ano 1, do ano } 2 \text { ao } 10 \text { aumento de } 2,7 \% \\
\text { ao ano. }\end{array}$ \\
$\begin{array}{c}\text { R } \$ 1,03 \text { no ano } 1, \text { do ano } 2 \text { ao } 10 \text { aumento de } 4,5 \% \\
\text { ao ano. }\end{array}$ \\
Revista da Universidade Vale do Rio Verde, Três Corações, v. 14, n. 1, p. 930-946, jan./jul. 2016
\end{tabular}


Rendimento Biodiesel (B20)

Consumo do Óleo Diesel

Geração de Glicerina

Poluição CO2 Óleo Diesel

Redução CO2 (B20)

Potencial poluidor por litro de óleo descartado de forma inadequada

Fonte: Próprios Autores (2015)

\subsubsection{Cenários Simulados}

Será modelado 3 cenário neste artigo, baseado nas informações coletas de especialistas e representantes da área de coleta de resíduos sólidos. Para uma melhor compreensão dos valores compreendidos em cada cenários apresenta-se a tabela 2, composta pelos valores totais de combustíveis gastos na simulação, a tabela apresenta também os 3 cenários simulados no trabalho, junto com a
94\% do rendimento do óleo diesel comum.

$3,03 \mathrm{Km} / \mathrm{l}$.

Fixada em $10 \%$.

2,669 $\mathrm{KgCO} 2$.

15,38\% em relação ao Óleo Diesel.

18400 litros de água.

porcentagem de biodiesel composto de cada mistura.

A partir destes 3 cenários, ficará exposta as diferenças econômicas, fatores denegridores ao meio ambiente e também capacidade de reutilização de um produto descartado, transformando-o em matéria prima novamente, estes valores serão anexadas nas variáveis correspondentes.

Tabela 2. Valores totais utilizados nos Cenários

\begin{tabular}{lcccc}
\hline & \% Diesel & \%Biodiesel & Óleo Utilizado (litros) & Diesel Utilizado (litros) \\
\hline Cenário Atual & 95 & 5 & 75.000 & 1.140 .000 \\
Cenário B10 & 90 & 10 & 150.000 & 1.108 .000 \\
Cenário B20 & 80 & 20 & 300.000 & 96.000 \\
\hline
\end{tabular}

Fonte: Próprios Autores(2016)

\section{Resultados}

Nesta seção serão apresentados os resultados da modelagem referentes à simulação aplicada neste artigo, realizou-se a criação de uma proposta para à reutilização como matéria prima do óleo de cozinha queimado.
Para realizar a análise dos resultados gerados pelo modelo de simulação, levou-se em consideração a combinação de uso do biodiesel com o diesel, com o propósito de ser utilizado nos 7 veículos que realizam a coleta de resíduos urbanos em alguns municípios da região central do estado do Rio Grande do Sul, a tabela 3 representa os 
valores anuais e mensais dos componentes

utilizados na simulação.

Tabela 3. Valores Utilizados na Simulação

\begin{tabular}{lcccc}
\hline & Óleo Anual & Diesel Anual & Óleo Mensal & Diesel Mensal \\
\hline Cenário Atual (litros) & 7.500 & 114.000 & 625 & 9.500 \\
Cenário B10 (litros) & 15.000 & 108.000 & 1.250 & 9.000 \\
Cenário B20 (litros) & 30.000 & 96.000 & 2.500 & 8.000 \\
\hline
\end{tabular}

Fonte: Próprios Autores(2016)

No período simulado, verificou-se uma grande diferença na emissão de $\mathrm{CO} 2$, a figura 7 apresenta os resultados dos 3 cenários simulados, o CenárioAtual gera cerca de 1.448 toneladas de $\mathrm{CO} 2$ ao ano enquanto o cenário positivo ao meio ambiente, o CenárioB20 gera cerca de 672toneladas de
$\mathrm{CO} 2$ ao ano, uma diferença de aproximadamente $53,36 \%$ no ano 10 . O CenárioB10 possui um potencial poluidor cerca de $32,75 \%$ menor quando comparado ao CenárioAtual, já que o CenárioB10 emite em média cerca de 974 toneladas ao ano.

Figura 7. Geração de $\mathrm{CO} 2$

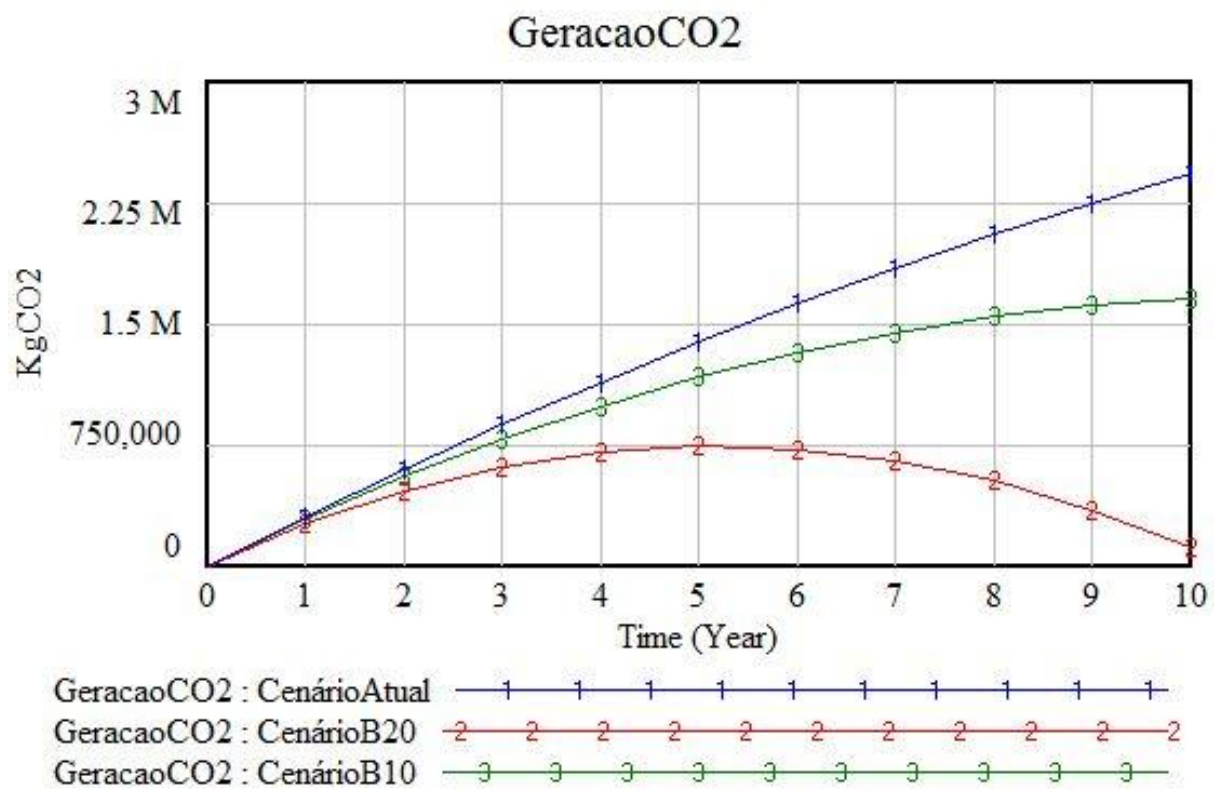

Fonte: Próprios Autores (2016) 
Nos cenários modelados levou-se em consideração a reciclagem do óleo de cozinha, verificando o seu impacto ambiental. A figura 8 apresenta a variação da poluição da água, evento normalmente causado pelo descarte inapropriado do óleo queimado, tendo em vista o melhor cenário para o meio ambiente, nota-se o cenárioB20, apresentando uma redução da poluição em aproximadamente 3 bilhões de litros ao ano, quando comparado ao pior cenário (CenárioAtual), possui $75 \%$ (cerca de 22 bilhões de litros de água) de diminuição na poluição da água ao final do ano 10. Verifica-se o cenárioB10 com uma redução de água de aproximadamente $50 \%$ menor à redução contida no CenárioAtual.

Figura 8. Redução da poluição da água

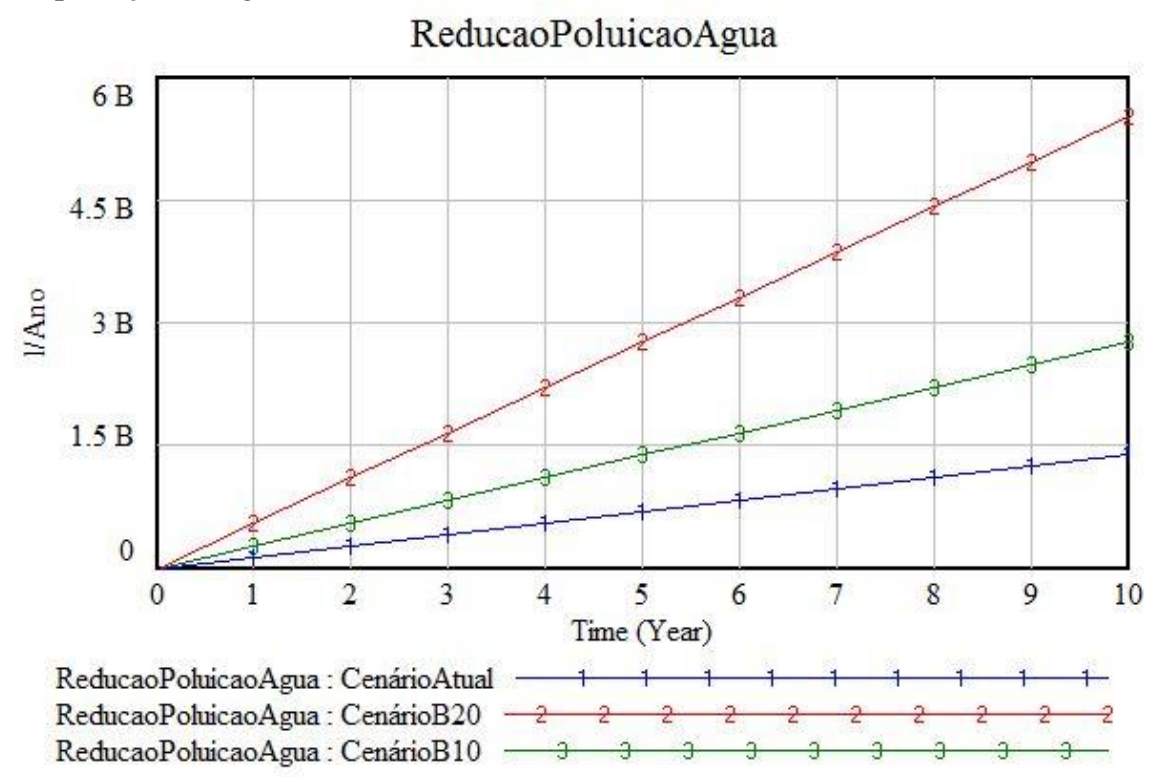

Fonte: Próprios Autores (2016)

A reutilização do óleo de cozinha no cenário $\mathrm{B} 20$, gera uma economia de mais de $\mathrm{R} \$$ 13.949 ao mês, já subtraídos os custos envolvidos na produção do biodiesel, pode-se verificar na figura 5 o crescimento da diferença da economia entre o Cenário B20 e o CenárioAtual, totalizando no ano 10 uma diferença de cerca de $44,41 \%$. Nota-se através da figura $9, \quad$ o cenário B20 apresentando uma melhoria considerável na economia de aproximadamente $7,45 \%$ ao ano. A Tabela 4 sumariza os resultados para ambos os cenários, demonstrando a crescente no valor gasto pela coleta total durante estes 10 anos. 
Figura 9. Economia gerada pelo modelo proposto

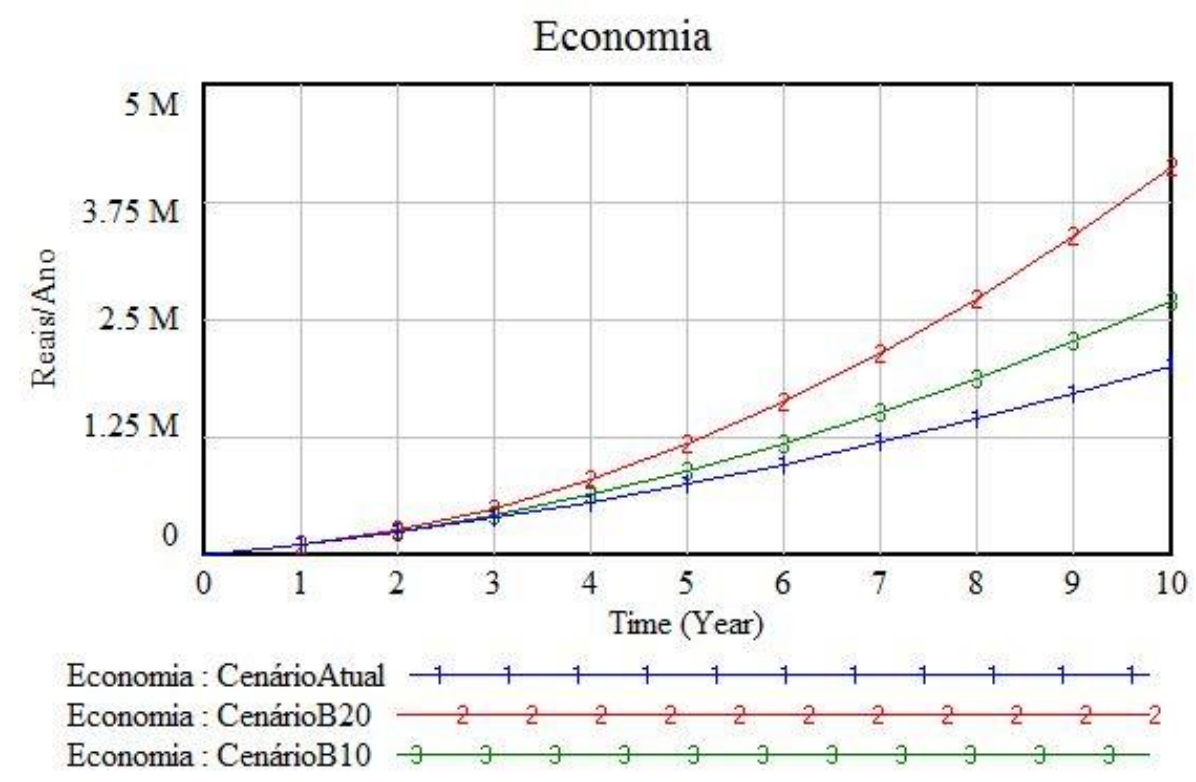

Fonte: Próprios Autores (2016)

\section{Validação do Modelo}

O modelo foi validado em diferentes etapas, inicialmente foram utilizados dados retirados a partir de estudo em artigos científicos, manuais técnicos referentes à área da gestão de resíduos sólidos e, também, obtidos com a participação de especialistas na área de gestão de resíduos, aqui fica caracterizada a validação nominal, visto que serão utilizados especialistas para definirem as variáveis importantes na modelagem proposta. Seguindo em frente, na segunda fase de validação, quanto da implementação no simulador Vensim (VENTANA SYSTEMS, 2011), serão utilizados dados atuais sobre a coleta de RSU na região central do estado do Rio Grande do Sul, com o objetivo de verificar a integração entre todas as variáveis compostas no modelo, bem como dos resultados gerados, já que serão avaliadas as saídas produzidas pelo modelo de simulação a partir de dados reais fornecidos.

Na ultima fase da validação, para a construção do experimento, serão utilizados dados e taxas reais referentes à utilização de biodiesel, e também taxas referentes ao aumento do valor do Diesel segundo o site www.globo.com. Para tal, serão gerados cenários a serem simulados no modelo. O detalhamento e quantificação de taxas para cada cenário simulado são apresentados a seguir na Seção 6.

\section{Considerações Finais}

Os resultados apresentados foram obtidos através de simulações feitas utilizando o software Vensim. Porém, já é possível afirmar que além da redução o impacto ambiental a economia gerada para um cenário de 10 anos, 
justifica a aplicação dos resultados gerados pelo modelo.

Dentre os principais resultados ocasionados pela reciclagem do óleo de cozinha tem-se uma economia de mais de $\mathrm{R} \$ 7$ milhões na aquisição de diesel, se o mesmo for misturado à uma porção de $20 \%$ de biodiesel , já subtraídos os custos envolvidos na produção do biodiesel. Quanto à redução dos impactos ambientais, também objeto de estudo da pesquisa, verificou-se que com a reciclagem do óleo e, com seu posterior uso nos veículos de coleta, a emissão de $\mathrm{CO} 2$ no meio ambiente seria reduzida em $7.761 .173 \mathrm{Kg}$, uma diminuição de 53\% aproximadamente, caso seja utilizada a maior mistura de biodiesel possível..

Quanto à redução da poluição da água, provou-se que cerca de 22 bilhões de litros de água não serão poluídos, caso $20 \%$ de Biodiesel seja misturado ao combustível utilizado na coleta dos resíduos sólidos urbanos . A Tabela 4 sumariza os cenários.

Tabela 4: Resultados sumarizados nos cenários estudados.

\begin{tabular}{cccc}
\hline & Cenário & Cenário & Cenário \\
& Atual (10 anos) & B10 (10 anos) & B20 (10 anos) \\
\hline Economia (Reais) & 0 & 2.478 .429 & $7.435 .275,30$ \\
Geração CO2 (Kg) & 14.488 .658 & 9.742 .911 & $6.727 .485,00$ \\
Red. Poluição Água (Litros) & 0 & 7.590 .000 .000 & 22.770 .000 .000 \\
\hline
\end{tabular}

Fonte: Próprios Autores (2016)

\section{Referências}

Abiove. Associação Brasileira das Indústrias de Óleos Vegetais. 2012. Disponível em: < http://www.abiove.org.br/>. Acesso em: 15 julho 2015.

Agarwal, A. K.; Das, L. M. "Biodiesel development and characterization for use as fuel in compression ignition engines".J. Eng. GasTurb. Power-T. ASME, v.123, p.440-447, 2001.

Barbosa, G. N.; Pasqualetto, A. "Aproveitamento do óleo residual de fritura na produção de Biodiesel", 2006. Trabalho Final de Graduação Universidade Católica de Goiás - Departamento de Engenharia - Engenharia Ambiental. Goiania Goiás. Disponível em: . Acesso em: 15 julho 2015.

Biodiesel. "Reciclagem de óleo de cozinha". Disponível em: <www.biodieselbr.com>.Acessado em março de
2008. BRASIL. Resolução $n^{\circ} 275$, de 25 de abril de 2001 do Conselho Nacional do Meio Ambiente (CONAMA).

BIODISELBR. Disponível em: <http://www.biodieselbr.com/biodiesel> . Acesso em: 15 julho de 2015.

BNDES - Banco Nacional do Desenvolvimento."Nova diretoria do BNDES lança programa do biodiesel", 2004. Disponível em: <http://www.bndes.gov.br/>. Acessado em Dez. 2014.

CONAMA - Conselho Nacional do MeioAmbiente .Resolução No 430, Brasília-DF, Maio 2011.

Cover, J. "Introduction to System Dynamics". Powersim Press, 1996.

Dace, Elina ; Bazbauers, G ; Berzina, A ; Davidsen. $\mathrm{P}$ "System dynamics model for analyzing effects of eco-design policy 
onpackaging waste management system", Resources, Conservation and Recycling 2014.

Deaton, M. L.; Winebrake, J. J.” Dynamic Modelling of Environmental Systems". SpringerVerlag, 2000.

Dorado, M. P.; Arnal, J. M.; Gomez, J.; Gil, A.; Lopez, F. J. The effect of waste vegetable oil blend with diesel fuel on engine performance. Trans. ASAE, v.45, p.525-529, 2002

Ferrari, A. R; Oliveira, V. S.; Seabio, A. Biodiesel de soja: taxa de conversão em ésteres etílicos, caracterização físico-química e consumo em gerador de energia. Química Nova, v.28, n.1, p.19-23, 2005

Ford, A. "Modeling the environment", Second Edition.Island Press, 2009.

Law, A. M.; Kelton, W. D. "Simulation Modeling \& Analysis".2nd ed. McGraw-Hill, 1991.

Oliveira , B. M. G ; Sommerlatte, B. R. (2009), "Plano de Gerenciamento Integrado do Resíduo óleo de cozinha", , Dísponivel em : $<$ http://www.projetoreciclar.ufv.br/docs/cartilha/p gi_oleo_cozinha.pdf>, acessado em mai. 2015

Oliveira,G.S.; Filho, R. D. O. "Análise do consumo de combustível de ônibus urbano", Anais doXVIII Congresso de Pesquisa e Ensino em Transportes (ANPET), 2014.

Mendes, P.A.S, 2015. "Sustentabilidade na produção e uso do Biodiesel”. Annriss, v.1, p67132, 2015.

Santos, A.M;Matal, P,H.L.S, "Aspectos técnicos e ambientais relativos ao uso de biodiesel em motores de combustão"2006, disponível em: http://www.interfacehs.sp.senac.br/BR/artigos.asp ?ed=6\&cod_artigo=108, acesso maio de 2015 .

Simonetto, E. de O. ;Lobler, M. L. "Simulação computacional para avaliação de cenários sobre a reciclagem de resíduos sólidos urbanos e o seu impacto na economia de energia".Anais do IX Simpósio Brasileiro de Sistemas de Informação. Universidade FUMEC, 2013.

Simonetto, E. O.; Rodrigues, G.O ;Dalmolin, L. C.; Modro, N. "O uso da dinâmica de sistemas para avaliação de cenários da reciclagem de resíduos sólidos urbanos", GEINTEC - Gestão, Inovação e Tecnologias, 4(2), 2014.

Toppan.J.E.N;Darbello.A.M , "Biodiesel", $17^{\circ}$ Congresso Brasileiro de Transporte e Trânsito, EMDEC, 2009.

VENSIM - Ventana Simulations (2014), Vensimsimulation software. Disponível em:<http://www.vensim.com>, 2014.

AcessadoemDez. 2014.

\section{VENTANA SYSTEMS. “Vensim Simulation} Software”. Disponível em:

<http://www.vensim.com>. Acesso em: mar. 2015.

Zucatto, L. C. ;Welle, I. ; Silva, T. N. D."Cadeia reversa do óleo de cozinha: coordenação, estrutura e aspectos relacionais" RAE - Revista de Administração de Empresas, 53(5), 2013. 